\title{
Extra Investigation Might Be Necessary in High-Risk CT-Negative Cerebrovascular Cases
}

\author{
Mohammad Sami Walid ${ }^{1}$, Nadezhda Zaytseva ${ }^{2}$ \\ ${ }^{1}$ Kingsbrook Jewish Medical Center, Brooklyn, USA; ${ }^{2}$ Department of Physical Education, Krasnodar State Medical University, \\ Krasnodar, Russia. \\ Email: mswalid@yahoo.com
}

Received February $21^{\text {st }}, 2013$; revised March $4^{\text {th }}, 2013$; accepted May $4^{\text {th }}, 2013$

Copyright (C) 2013 Mohammad Sami Walid, Nadezhda Zaytseva. This is an open access article distributed under the Creative Commons Attribution License, which permits unrestricted use, distribution, and reproduction in any medium, provided the original work is properly cited.

\begin{abstract}
Cerebrovascular accidents occur in elderly patients in whom neurodegenerative changes increase the risk of vascular permeation and concomitant intracranial bleeding. In rare cases, intracerebral massive hemorrhage happens in hospitalized patients with apparently stable clinical picture. We report two cases illustrating this phenomenon, discuss the possible pathomechanism involved in those tragic incidents and suggest possible prevention measures for these patients. A negative CT image in a patient with neurological symptoms, especially who are on warfarin with or without considerably elongated coagulation time, may be subject to a sudden intracranial hemorrhage. Microvascular abnormalities might be detected on perfusion CT imaging and should therefore be performed in all CT negative patients with close observation for 24 hours before contemplating discharge.
\end{abstract}

Keywords: Subdural Hematoma; Intracerebral Hemorrhage; Warfarin; Perfusion CT

\section{Introduction}

Cerebrovascular accidents occur in elderly patients in whom neurodegenerative changes increase the risk of vascular permeation and concomitant intracranial bleeding. Microvascular brain changes accelerate cognitive decline in patients with already diminished cognitive capacities. However, intracranial hemorrhage if massive and bilateral often leads to quick death. In such cases, emergency evacuation is indicated to save the patient's life. Unfortunately, in rare cases, intracerebral massive hemorrhage happens in hospitalized patients with apparently stable clinical picture. In this paper, we report two cases illustrating this phenomenon, discuss the possible pathomechanism involved in those tragic incidents and suggest possible prevention measures for these patients.

\section{Case 1}

A 77-year-old Caucasian male with multiple chronic health problems including end-stage renal disease on hemodialysis, congestive heart failure with coronary artery disease, Parkinson's disease, dementia, and chronic atrial fibrillation was admitted with confusion and rightsided weakness. He had multiple falls in the past and a history of an intracranial bleeding four years ago (Figure 1(A)) when he was on warfarin. His prothrombin time (PT) was 16.1 and INR 1.25. This was subsequently discontinued. The patient had a couple more falls lately and then became less awake and poorly responsive. Computed tomography (CT) showed a $9 \times 4 \mathrm{~cm}$ subdural fluid collection in the left parietal lobe with significant compression and some midline shift (Figure 1(B)). After the patient had dialysis he underwent craniotomy. The dura was opened and immediately there was substantial extra axial fluid extrusion compatible with a chronic and acute subdural hematoma. The brain; however, did not expand. He developed respiratory failure and was placed on ventilator. He needed a second craniotomy and drainage because of rebleeding. Next day brain CT showed a marked intraparenchymal hemorrhage and associated subarachnoid hemorrhage in the left frontal, temporal and parietal lobes (Figure 1(C)). The size of the brain hematoma was $7.6 \times 5.4 \mathrm{~cm}$. There was increased edema, mass effect and subfalcine herniation $12 \mathrm{~mm}$ of midline shift from left to right. The patient became unstable. His family opted to have palliative care involved and to do care and comfort only. The patient expired shortly after. 


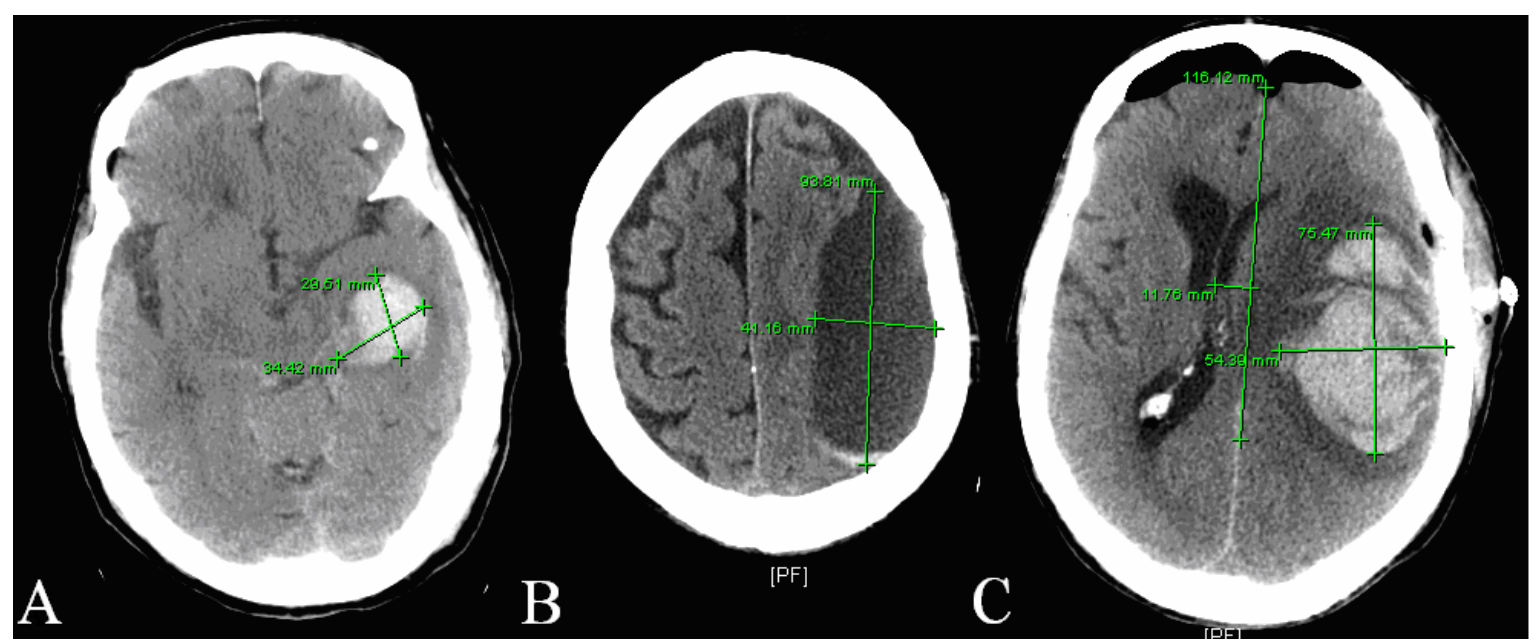

Figure 1. (A) 4-year old CT of the brain showing left intracerebral hemorrhage; (B) Current CT showing a huge left subdural hematoma; (C) Current CT showing a huge left intracerebral hematoma.

\section{Case 2}

A 68-year-old white male presented to the emergency room at noon with acute onset of left-sided weakness. The patient had a history of congestive heart failure secondary to cardiomyopathy and he had an ejection fraction of $25 \%$ and was on chronic warfarin therapy for atrial fibrillation. He had had a pacemaker placed about 2 months before and had not been recuperating well since that time. The patient had been complaining of worsening shortness of breath and increasing anxiety. He was seen by his primary cardiologist prior to admission and was instructed to increase his furosemide. On the night of admission, the patient started having some left-sided weakness and was unable to ambulate. His wife called 911 and the patient was brought to the emergency room for further evaluation. On evaluation in the emergency department the patient's examination was pretty benign, blood pressure was normal. He was alert and able to speak. He stated he had sudden onset of left-sided weakness and some slurred speech. Motor strength was good and he appeared to be appropriate mentally. The coagulation profile was as follows: PT 53.7 (Ref. range 12.3 15.2), INR 5.75. Warfarin was held, fresh frozen plasma and vitamin $\mathrm{K}$ were given. The patient was admitted for transient ischemic attack. His brain CT scan was read as normal (Figure 2(A)). For chest pain, the patient was put on nitroglycerin patch.

The next morning, the patient had an episode of vomiting and became unresponsive. The patient was intubated and transferred to the intensive care unit. Another CT scan was performed which showed a new $4 \mathrm{~cm}$ left parietal intracerebral hematoma (Figure 2(B)). There was also new hypodensity in the gray and white matter structures above the ventricular level bilaterally. The patient's condition continued to deteriorate and on the

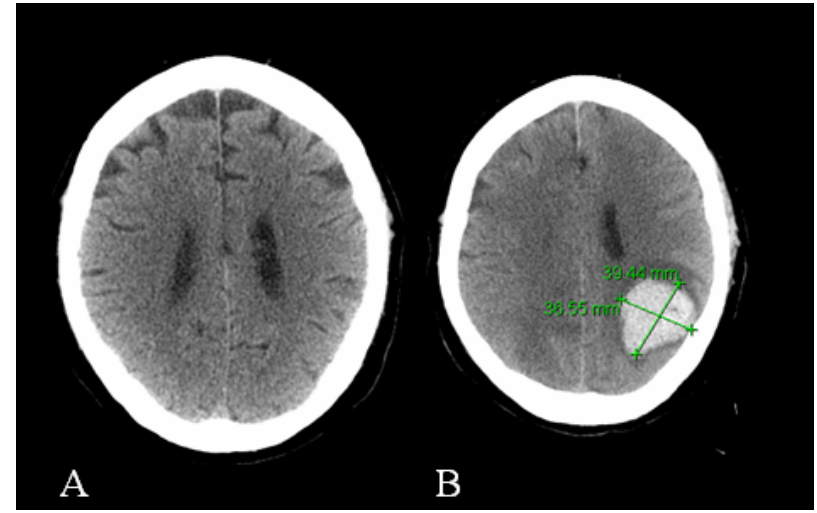

Figure 2. CT imaging without contrast during admission to hospital (A) and the next morning in hospital (B) showing a negative first $\mathrm{CT}$ scan and a large left parietal intracerebral hemorrhage on the second CT scan.

second hospital day repeat CT showed significant worsening of diffuse cerebral edema and mass effect in the posterior fossa with downward herniation. After discussion with the family nuclear brain flow study was ordered. The patient had negative brain flow study with no tracer in the brain, suggestive of intracranial death. The patient was extubated and expired shortly thereafter.

\section{Discussion}

Subdural hematomas often occur in elderly patients in which neurodegenerative changes increase the risk of uncontrolled falls and concomitant head trauma. Such hematomas may be massive and bilateral and if untreated negatively affect cognitive function in patients with already diminished cognitive capacities. Therefore, evacuation is indicated and mainly required to improve cognitive outcome. Unfortunately, in rare cases a secondary intracranial hemorhage happens after evacuation of 
a primary subdural hematoma. This may occur as a new subdural hematoma on the other side or an intracerebral hematoma on either side or in the brainstem or even as an epidural hematoma [1-11]. Postoperative intracerebral hemorrhage is rare and estimated at $0.5 \%-2 \%[4,5$, 11-13]. Early surgical evacuation of the mass lesion is essential for a favorable outcome, but the severity of the underlying brain injury determines the outcome, even when surgery has been prompted [14].

Prolonged brain retraction may cause severe disturbance in the parenchymal biochemical patterns in the brain underlying an evacuated acute subdural hematoma leading to microthrombosis or vascular wall disintegration [14]. This combined with defective autoregulation and hyperemic long-lasting regional cerebral blood flow values can cause secondary bleeding leading to unfavorable outcome $[2,8,10,15,16]$. Bleeding has also been reported during the evacuation process [17].

Our patient had multiple systemic disease and a history of intracerebral hemorrhage on the same side four years ago and subsequent discontinuation of antithrombotic treatment. This fact seems to be related to the fatal outcome of this case. Previous cerebrovascular incidents may form internal weak spots which may converge with external circumstances (evacuation/pressure relief) resulting in a massive intracerbral bleeding and death [5, 10]. Therefore, perfusion CT may be necessary before evacuating a large subdural hematoma to differentiate salvageable ischemic penumbra from unsalvageable infarcted brain tissue under the hematoma [18]. This may help identify patients most likely to benefit from hematoma evacuation and decrease the risk of secondary inctracerebral bleeding. In addition, evacuation of a large subdural hemtoma should be performed slowly with extreme caution in frail patients. There may be as well an argument for partial evacuation in these patients.

The incidences of major bleeding and intracranial hemorrhages with standard-dose warfarin are known to occur at a rate of $1.6 \%$ and $2.5 \%$, respectively [19]. Delayed expansion is known in warfarin-treated patients who present with intracerebral hemorrhages [20-22]; however, it is rare that a patient on warfarin presents with symptoms of neurovascular accident and negative computed tomography (CT) imaging and after many hours develops a large intracerebral hemorrhage and dies.

The second case illustrates the possibility of symptom-image dissociation in stroke patients. A negative CT image in a patient with neurological symptoms, especially those who are on warfarin with or without considerably elongated coagulation time, may be subject to an imminent intracranial hemorrhage. Microvascular abnormalities might be detected on perfusion CT imaging which has been found to be useful for noninvasive diagnosis of cerebral ischemia and infarction including evaluation of vasospasm after subarachnoid hemorrhage [23]. Perfusion CT has also been used for assessment of cerebrovascular reserve in candidates for neurobypass surgery, neuroendovascular treatment, and for the evaluation of microvascular permeability in patients with intracranial neoplasms [23]. Perfusion CT should therefore be performed in all neurosymptomatic patients with CT-negative images to detect microvascular points of interest predisposing to sudden macrovascular incidents.

\section{REFERENCES}

[1] K. J. Park, et al., "Brain Stem Hemorrhage Following Burr Hole Drainage for Chronic Subdural HematomaCase Report,” Neurologia Medico-Chirurgica, Vol. 49, No. 12, 2009, pp. 594-597.

doi:10.2176/nmc.49.594

[2] S. Muneza, M. R. Rasoloherimampiononiaina and M. J. Nduwamariya, "Postoperative Intracerebral and Intraventricular Hemorrhages Following Removal of a Chronic Subdural Hematoma,” Journal of Clinical Neuroscience, Vol. 16, No. 10, 2009, pp. 1346-1348. doi:10.1016/j.jocn.2008.12.020

[3] S. G. Kannuki, N. Shirakawa and H. Toi, "Rapid Recurrence of Chronic Subdural Hematoma by CSF Entrapment,” No Shinkei Geka, Vol. 37, No. 6, 2009, pp. 567-572.

[4] A. Akhaddar, et al., "Combined Epidural and Intracerebral Hematomas after Evacuation of Bilateral Chronic Subdural Hematoma," Neurochirurgie, Vol. 54, No. 6, 2008, pp. 728-730. doi:10.1016/j.neuchi.2008.09.001

[5] S. Ulivieri and G. Oliveri, "Intracerebral Haemorrhage Following Surgical Evacuation of Chronic Subdural Haematoma: Case Report,” G Chir, Vol. 29, No. 5, 2008, pp. 233-234.

[6] C. Dinc, et al., "Intracerebral Haemorrhage Occurring at Remote Site Following Evacuation of Chronic Subdural Haematoma," Acta Neurochirurgica, Vol. 150, No. 5, 2008, pp. 497-499. doi:10.1007/s00701-008-1512-2

[7] A. Moussaoui, et al., "Spontaneous Intracerebral Haemorrhage Following Evacuation of Chronic Subdural Haematoma," Annales Françaises d'Anesthésie et de Réanimation, Vol. 25, No. 4, 2006, pp. 468-469. doi:10.1016/j.annfar.2005.11.007

[8] J. Sousa, et al., "Spontaneous Intracerebral Haemorrhage Following Evacuation of Chronic Subdural Hematomas," Journal of Clinical Neuroscience, Vol. 11, No. 7, 2004, pp. 794-796. doi:10.1016/j.jocn.2003.12.003

[9] S. V. Basulto, et al., "Postoperative Intracerebral Hematoma. An Unusual Complication of Chronic Subdural Hematoma,” Revista de Neurología, Vol. 38, No. 5, 2004, pp. 497-498.

[10] P. Diaz and A. Maillo, "Intracerebral Hemorrhage Following Chronic Subdural Hematoma Evacuation: Report of Two Cases and Review of the Literature," Neurocirugia (Astur), Vol. 14, No. 4, 2003, pp. 333-336.

[11] P. Missori, et al., "Intraparenchymal Haemorrhage after Evacuation of Chronic Subdural Haematoma. Report of 
Three Cases and Review of the Literature," British Journal of Neurosurgery, Vol. 16, No. 1, 2002, pp. 63-66. doi:10.1080/026886902753512637

[12] V. Rohde, G. Graf and W. Hassler, "Complications of Burr-Hole Craniostomy and Closed-System Drainage for Chronic Subdural Hematomas: a Retrospective Analysis of 376 Patients,” Neurosurgical Review, Vol. 25, No. 1-2, 2002, pp. 89-94. doi:10.1007/s101430100182

[13] P. Pencalet, “Complications of Chronic Subdural Hematoma in the Adult," Neurochirurgie, Vol. 47, No. 5, 2001, pp. 491-494.

[14] R. Hlatky, et al., "Evolution of Brain Tissue Injury after Evacuation of Acute Traumatic Subdural Hematomas," Neurosurgery, Vol. 55, No. 6, 2004, pp. 1318-1323. doi:10.1227/01.NEU.0000143029.42638.2C

[15] A. Chieregato, et al., "Hyperemia Beneath Evacuated Acute Subdural Hematoma Is Frequent and Prolonged in Patients with an Unfavorable Outcome: A Xe-Computed Tomographic Study,” Neurosurgery, Vol. 64, No. 4, 2009, pp. 705-717. doi:10.1227/01.NEU.0000341872.17024.44

[16] T. Sakaki, et al., "Delayed Intracerebral Haemorrhage after Intracranial Surgery,” Journal of Clinical Neuroscience, Vol. 6, No. 1, 1999, pp. 54-57. doi:10.1016/S0967-5868(99)90607-0

[17] M. Sato, et al., "Intracerebral Haemorrhage during Surgery for Chronic Subdural Haematoma,” Journal of Clinical Neuroscience, Vol. 14, No. 1, 2007, pp. 81-83. doi:10.1016/j.jocn.2005.12.044

[18] Y. W. Lui, et al., "Evaluation of CT Perfusion in the Set- ting of Cerebral Ischemia: Patterns and Pitfalls,” American Journal of Neuroradiology, Vol. 31, 2010, pp. 15521563.

[19] S. Suzuki, T. Yamashita, T. Kato, T. Fujino, K. Sagara, H. Sawada, T. Aizawa and L. T. Fu, "Incidence of Major Bleeding Complication of Warfarin Therapy in Japanese Patients with Atrial Fibrillation,” Circulation Journal, Vol. 71, No. 5, 2007, pp. 761-765. doi:10.1253/circj.71.761

[20] J. J. Flibotte, N. Hagan, J. O’Donnell, S. M. Greenberg and J. Rosand, "Warfarin, Hematoma Expansion, and Outcome of Intracerebral Hemorrhage,” Neurology, Vol. 63, No. 6, 2004, pp. 1059-1064. doi:10.1212/01.WNL.0000138428.40673.83

[21] S. B. Lee, E. M. Manno, K. F. Layton and E. F. Wijdicks, "Progression of Warfarin-Associated Intracerebral Hemorrhage after INR Normalization with FFP,” Neurology, Vol. 67, No. 7, 2006, pp. 1272-1274. doi:10.1212/01.wnl.0000238104.75563.2f

[22] M. Yasaka, K. Minematsu, H. Naritomi, T. Sakata and T. Yamaguchi, "Predisposing Factors for Enlargement of Intracerebral Hemorrhage in Patients Treated with Warfarin,” Journal of Thrombosis and Haemostasis, Vol. 89, 2003, pp. 278-283.

[23] E. G. Hoeffner, I. Case, R. Jain, S. K. Gujar, G. V. Shah, J. P. Deveikis, R. C. Carlos, B. G. Thompson, M. R. Harrigan and S. K. Mukherji, "Cerebral Perfusion CT: Technique and Clinical Applications," Radiology, Vol. 231, No. 3, 2004, pp. 632-644. doi:10.1148/radiol.2313021488 